\title{
VIABILITY OF A TIME DEPENDENT CLOSED SET WITH RESPECT TO A SEMILINEAR DELAY EVOLUTION INCLUSION*
}

\author{
BY
}

\author{
MIHAI NECULA and MARIUS POPESCU
}

\begin{abstract}
We prove a sufficient condition for a time-dependent closed set to be viable with respect to a delay evolution inclusion governed by a strongly-weakly u.s.c. perturbation of an infinitesimal generator of a $C_{0}$-semigroup. This condition is expressed in terms of a natural concept involving tangent sets, generalizing tangent vectors in the sense of Bouligand and Severi.
\end{abstract}

Mathematics Subject Classification 2010: 34K09, 34G25, 34A60, 47D06.

Key words: differential delay evolution inclusion, locally closed graph, tangent set, tangency condition, multi-valued mapping, viability.

\section{Introduction}

Let $X$ be a real Banach space, $I=[a, b) \subseteq \mathrm{R}$ be a nonempty and bounded interval and let $A: D(A) \subseteq X \rightarrow X$ be the infinitesimal generator of a $C_{0^{-}}$ semigroup $\{S(t) ; t \geq 0\}$ of type $(M, \omega)$, i.e., $\|S(t)\| \leq M e^{\omega t}$ for each $t \geq 0$.

Let $\sigma>0$ and $C_{\sigma}=C([-\sigma, 0] ; X)$ endowed with the norm

$$
\|\psi\|_{\sigma}=\sup \{\|\psi(t)\| ; t \in[-\sigma, 0]\} .
$$

If $u \in C([\tau-\sigma, T], X)$, then for each $t \in[\tau, T]$ we denote by $u_{t} \in C_{\sigma}$ the function given by

$$
u_{t}(s)=u(t+s) \text { for } s \in[-\sigma, 0] .
$$

\footnotetext{
*Supported by a grant of the Romanian National Authority for Scientific Research, CNCS-UEFISCDI, project number PN-II-ID-PCE-2011-3-0052.
} 
Let $K: I \rightsquigarrow X$ and $F: \mathcal{K} \rightsquigarrow X$ be two multi-functions with nonempty values, where

$$
\mathcal{K}=\left\{(t, \psi) \in I \times C_{\sigma} ; \psi(0) \in K(t)\right\} .
$$

Our aim here is to prove some new necessary and sufficient conditions in order that $\mathcal{K}$ be viable with respect to $A+F$.

To be more precise, let $(\tau, \varphi) \in \mathcal{K}$ and consider the Cauchy Problem

$$
\left\{\begin{array}{l}
u^{\prime}(t) \in A u(t)+F\left(t, u_{t}\right), \quad t \geq \tau \\
u_{\tau}=\varphi
\end{array}\right.
$$

Definition 1.1. By a mild solution of (1.1) on $[\tau, T] \subseteq I$, we mean a function $u \in C([\tau-\sigma, T] ; X)$ satisfying $\left(t, u_{t}\right) \in \mathcal{K}$ for $t \in[\tau, T], u(t)=$ $\varphi(t-\tau)$ for $t \in[\tau-\sigma, \tau]$ and for which there exists $f \in L^{1}(\tau, T ; X)$ with $f(t) \in F\left(t, u_{t}\right)$ a.e. for $t \in[\tau, T]$ and

$$
u(t)=S(t-\tau) \varphi(0)+\int_{\tau}^{t} S(t-s) f(s) d s
$$

for each $t \in[\tau, T]$.

Definition 1.2. We say that $\mathcal{K}$ is mild viable with respect to $A+F$, where $F: \mathcal{K} \rightsquigarrow X$, if for each $(\tau, \varphi) \in \mathcal{K}$, there exists $T>\tau$, such that $[\tau, T] \subseteq I$ and (1.1) has at least one mild solution $u:[\tau-\sigma, T] \rightarrow X$. If $T \in(\tau, \sup I)$ can be taken arbitrary, we say that $\mathcal{K}$ is globally mild viable with respect to $A+F$.

The existence of solutions for functional differential equations governed or not by linear and nonlinear operators in Banach spaces has been studied extensively in many papers.

The first viability results for (1.1) in the case $A=0$ and $F$ single valued have been proved in the papers LAKSHMIKANTHAM, LEELA and Moauro [11] and Leela and Moauro [12]. The case when $A=0, X$ is a finite dimensional space and $F$ is upper semicontinuous and with convex compact values has been studied by HADDAD $[9,10]$. The Haddad's result has been extended by Gavioli and Malaguti [8] to the infinite dimensional setting. The case when $A$ is the infinitesimal generator of $C_{0^{-}}$ semigroup and $F$ is a continuous single-valued function has been studied by PAVEL and IACOB [17] and the corresponding multivalued case, with $F$ 
a Carathèodory multifunction with nonconvex values, has been considered by Lupulescu and Necula [13].

As concern the case of delay evolution inclusions subjected to nonlocal initial conditions, a very recent research direction, we mention here the results established by VRabie [20, 21, 22, 23], Burlic $\breve{A}$ and Roşu [2] and

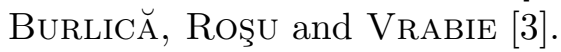

In this paper we shall use a concept of tangent sets that extends the notion of tangent vectors in the sense of Bouligand and Severi. This concept was introduced, in the specific case in which $K$ does not depend on $t$, by CÂRJĂ, Necula and Vrabie $[4,5]$, and it was adapted for the $t$-dependent case in Necula, Popescu and Vrabie $[15,16]$.

\section{Tangency concepts}

If $(Y, d)$ is a metric space, $y \in Y$ and $r>0, D(y, r)$ denotes the closed ball with center $y$ and radius $r>0$, i.e. $D(y, r)=\{x \in Y ; d(y, x) \leq r\}$,

while $S(y, r)$ denotes the open ball with center $y$ and radius $r>0$, i.e. $S(y, r)=\{x \in Y ; d(y, x)<r\}$. If $B \subseteq Y$ and $C \subseteq Y$, we denote by

$$
\operatorname{dist}(y, C)=\inf \{d(y, z) ; z \in C\}
$$

and by

$$
\operatorname{dist}(B, C)=\inf \{d(x, y) ; x \in B, y \in C\} .
$$

Also $\mathcal{B}(Y)$ denotes the family of all nonempty and bounded subsets of $Y$.

Definition 2.1. Let $Y \subseteq X$ be nonempty. The function $\beta_{Y}: \mathcal{B}(X) \rightarrow$ $\mathbb{R}_{+}$, defined by

$$
\beta_{Y}(B):=\inf \left\{\varepsilon>0 ; \exists x_{1}, x_{2}, \ldots, x_{n(\varepsilon)} \in Y, B \subseteq \bigcup_{i=1}^{n(\varepsilon)} D\left(x_{i}, \varepsilon\right)\right\},
$$

is called the Hausdorff measure of noncompactness on $X$ subordinated to $Y$. If $Y=X$, we simply denote $\beta_{X}$ by $\beta$, and we simply call it the Hausdorff measure of noncompactness on $X$.

Remark 2.1. We have the following properties:

(i) for each $B \in \mathcal{B}(X)$ and $r>0$ with $B \subseteq D(0, r)$, we have $\beta(B) \leq r$;

(ii) $\beta(B)=0$ if and only if $B$ is relatively compact; 
(iii) the restriction of $\beta_{Y}$ to $\mathcal{B}(Y)$ coincides with the Hausdorff measure of noncompactness on $Y$;

(iv) for each $B \in \mathcal{B}(Y)$ we have $\beta(B) \leq \beta_{Y}(B) \leq 2 \beta(B)$.

The next lemma is due to MÖNCH [14].

Lemma 2.1. Let $X$ be a separable Banach space and $\left\{f_{n} ; n \in \mathbb{N}\right\}$ a subset in $L^{1}(\tau, T ; X)$ for which there exists $\ell \in L^{1}\left(\tau, T ; \mathbb{R}_{+}\right)$such that

$$
\left\|f_{n}(s)\right\| \leq \ell(s)
$$

for each $n \in \mathbb{N}$ and a.e. for $s \in[\tau, T]$. Then the mapping

$$
s \mapsto \beta\left(\left\{f_{n}(s) ; n \in \mathbb{N}\right\}\right)
$$

is integrable on $[\tau, T]$ and, for each $t \in[\tau, T]$, we have

$$
\beta\left(\left\{\int_{\tau}^{t} f_{n}(s) d s ; n \in \mathbb{N}\right\}\right) \leq \int_{\tau}^{t} \beta\left(\left\{f_{n}(s) ; n \in \mathbb{N}\right\}\right) d s .
$$

For further details on the Haussdorf measure of noncompactness see CÂRJĂ, NeCUla and VRABIE [4], Section 2.7, pp. 48 53.

Definition 2.2. Let $(Y, d)$ be a metric space and let $F: Y \rightsquigarrow X$ a multi-function. We say that $F$ is u.s.c. at $y \in Y$ if for each open set $U \subseteq X$ with $F(y) \subseteq U$ there exists an open set $V \subseteq Y$ with $y \in V$ and $F(V) \subseteq U$. We say that $F$ is u.s.c. on $Y$ if it is u.s.c. at each $y \in Y$.

Throughout, $\mathcal{K}$ is endowed with the metric, $d$, defined by

$$
d((\tau, \varphi),(\theta, \psi))=\max \left\{|\tau-\theta|,\|\varphi-\psi\|_{\sigma}\right\},
$$

for all $(\tau, \varphi),(\theta, \psi) \in \mathcal{K}$. Furthermore, whenever we will use the term strongly-weakly u.s.c. we will mean that the domain of the multi-function in question is equipped with the strong topology, while the range is equipped with the weak topology. The term u.s.c. refers to the case in which both domain and range are endowed with the strong topology.

Definition 2.3. The multi-function $F: \mathcal{K} \rightsquigarrow X$ is called locally bounded if, for each $(\tau, \varphi) \in \mathcal{K}$, there exist $\delta>0, \rho>0$, and $M_{0}>0$ such that for all $(t, \psi) \in([\tau-\delta, \tau+\delta] \times D(\varphi, \rho)) \cap \mathcal{K}$, we have

$$
\|F(t, \psi)\| \leq M_{0} .
$$


Let $(\tau, \varphi) \in \mathcal{K}$, let $\eta \in X$ and let $E \in \mathcal{B}(X)$.

Definition 2.4. We say that

(i) $\eta$ is $A$-right-tangent to $\mathcal{K}$ at $(\tau, \varphi)$ if

$$
\liminf _{h \downarrow 0} \frac{1}{h} \operatorname{dist}\left(S(h) \varphi(0)+\int_{0}^{h} S(h-s) \eta d s, K(\tau+h)\right)=0 ;
$$

(ii) $E$ is $A$-right-tangent to $\mathcal{K}$ at $(\tau, \varphi)$ if

$$
\liminf _{h \downarrow 0} \frac{1}{h} \operatorname{dist}\left(S(h) \varphi(0)+\int_{0}^{h} S(h-s) E d s, K(\tau+h)\right)=0 ;
$$

(iii) $E$ is $A$-right-quasi-tangent to $\mathcal{K}$ at $(\tau, \varphi)$ if

$$
\liminf _{h \downarrow 0} \frac{1}{h} \operatorname{dist}\left(S(h) \varphi(0)+\int_{0}^{h} S(h-s) \mathcal{F}_{E} d s, K(\tau+h)\right)=0,
$$

where

$$
\mathcal{F}_{E}=\left\{f \in L_{\mathrm{loc}}^{1}(\mathbb{R} ; X) ; f(s) \in E \text { a.e. for } s \in \mathbb{R}\right\} .
$$

Throughout, we denote by:

(i) $\mathcal{T}_{\mathcal{K}}^{A}(\tau, \varphi)$ the set of all $A$-right-tangent vectors to $\mathcal{K}$ at $(\tau, \varphi)$;

(ii) $\mathcal{T}_{\mathcal{K}}^{A}(\tau, \varphi)$ the set of all $A$-right-tangent sets to $\mathcal{K}$ at $(\tau, \varphi)$;

(iii) ${ }_{2} \mathcal{T S}_{\mathcal{K}}^{A}(\tau, \varphi)$ the set of all $A$-right-quasi-tangent sets to $\mathcal{K}$ at $(\tau, \varphi)$.

If $K$ is constant, $E$ is $A$-right-tangent to $\mathcal{K}$ at $(\tau, \varphi)$ if and only if it is $A$ tangent to $K$ at $\xi=\varphi(0)$ in the sense of CÂRJĂ, NeCUla and VRABIE $[4,5]$, i.e., if and only if $E \in \mathcal{T S}_{K}^{A}(\xi)$, which means

$$
\liminf _{h \downarrow 0} \frac{1}{h} \operatorname{dist}\left(S(h) \xi+\int_{0}^{h} S(h-s) E d s, K\right)=0 .
$$

Similarly, if $K$ is constant, $E$ is right-quasi-tangent to $\mathcal{K}$ at $(\tau, \varphi)$ if and only if it is $A$-quasi-tangent to $K$ at $\xi=\varphi(0)$ in the sense of CÂRJ $\breve{A}$, Necula and VRabie $[4,5]$, i.e., if and only if $E \in \mathcal{Q T S}_{K}^{A}(\xi)$ ), which means

$$
\liminf _{h \downarrow 0} \frac{1}{h} \operatorname{dist}\left(S(h) \xi+\int_{0}^{h} S(h-s) \mathcal{F}_{E} d s, K\right)=0 .
$$

By identifying vectors with singleton sets and constants with locally integrable functions, we have

$$
\mathcal{T}_{\mathcal{K}}^{A}(\tau, \varphi) \subset \mathcal{T S}_{\mathcal{K}}^{A}(\tau, \varphi) \subset Q \mathcal{T} \mathcal{S}_{\mathcal{K}}^{A}(\tau, \varphi),
$$

and it may happen that, even in the simplest case $A \equiv 0$, both inclusions to be strict. See Example 2.4.1, p. 36 in CÂRJ $\breve{A}$, NeCula and VRABIE [4]. 


\section{Necessary conditions for viability}

For the proof of the main result of this section we need the following simple lemma:

Lemma 3.1. Let $f:[\tau, T] \rightarrow X$ be a measurable function and $B, C \subset X$ two nonempty sets such that $f(t) \in B+C$ a.e. for $t \in[\tau, T]$. Then, for every $\varepsilon>0$ there exist three measurable functions, $b:[\tau, T] \rightarrow B, c:[\tau, T] \rightarrow C$ and $r:[\tau, T] \rightarrow S(0, \varepsilon)$ such that

$$
f(t)=b(t)+c(t)+r(t) \text { a.e. for } t \in[\tau, T] .
$$

Proof. Let $\varepsilon>0$ be fixed. Let $\bar{f}:[\tau, T] \rightarrow X$ be countably valued and such that $\|\bar{f}(t)-f(t)\|<\frac{\varepsilon}{2}$ a.e. for $t \in[\tau, T]$. So, we have

$$
\bar{f}(t) \in B+C+S\left(0, \frac{\varepsilon}{2}\right) \text { a.e. for } t \in[\tau, T] .
$$

Then, there exist three countably valued functions $b:[\tau, T] \rightarrow B, c:$ $[\tau, T] \rightarrow C$ and $\bar{r}:[\tau, T] \rightarrow S\left(0, \frac{\varepsilon}{2}\right)$ such that

$$
\bar{f}(t)=b(t)+c(t)+\bar{r}(t) \text { a.e. for } t \in[\tau, T] .
$$

The proof is complete once we take $r(t)=\bar{r}(t)+f(t)-\bar{f}(t)$.

Theorem 3.1. If $F: \mathcal{K} \rightsquigarrow X$ is u.s.c. and $\mathcal{K}$ is mild viable with respect to $A+F$ then, for all $(\tau, \varphi) \in \mathcal{K}$ we have

$$
\lim _{h \downarrow 0} \frac{1}{h} \operatorname{dist}\left(S(h) \varphi(0)+\int_{0}^{h} S(h-s) \mathcal{F}_{F(\tau, \varphi)} d s, K(\tau+h)\right)=0 .
$$

Proof. Let $(\tau, \varphi) \in \mathcal{K}$ and $u:[\tau-\sigma, T] \rightarrow X$ be a mild solution of (1.1). Hence there exists $f \in L^{1}(\tau, T ; X)$ such that $f(s) \in F\left(s, u_{s}\right)$ a.e. for $s \in[\tau, T]$ and

$$
u(t)=S(t-\tau) \varphi(0)+\int_{\tau}^{t} S(t-s) f(s) d s
$$

for all $t \in[\tau, T]$.

Let $\varepsilon>0$ be arbitrary but fixed.

Since $F$ is u.s.c. at $(\tau, \varphi)$ and $\lim _{t \rightarrow \tau} u_{t}=u_{\tau}=\varphi$ in $C_{\sigma}$, we may find $\delta>0$ such that

$$
f(s) \in F\left(s, u_{s}\right) \subseteq F(\tau, \varphi)+S(0, \varepsilon) \text { a.e. for } s \in[\tau, \tau+\delta] .
$$


From Lemma 3.1, we deduce that there exist two integrable functions $g:[\tau, \tau+\delta] \rightarrow F(\tau, \varphi)$ and $r:[\tau, \tau+\delta] \rightarrow S(0,2 \varepsilon)$ such that $f(s)=$ $g(s)+r(s)$ a.e. for $s \in[\tau, \tau+\delta]$.

Since $u(\tau+h) \in K(\tau+h)$, we deduce that for each $0<h<\delta$

$$
\begin{aligned}
& \frac{1}{h} \operatorname{dist}\left(S(h) \varphi(0)+\int_{\tau}^{\tau+h} S(\tau+h-s) \mathcal{F}_{F(\tau, \varphi)} d s, K(\tau+h)\right) \\
& \leq \frac{1}{h} \operatorname{dist}\left(S(h) \varphi(0)+\int_{\tau}^{\tau+h} S(\tau+h-s) \mathcal{F}_{F(\tau, \varphi)} d s, S(h) \varphi(0)\right. \\
& \left.+\int_{\tau}^{\tau+h} S(\tau+h-s) f(s) d s\right) \\
& \leq \frac{1}{h} \operatorname{dist}\left(\int_{\tau}^{\tau+h} S(\tau+h-s) g(s) d s, \int_{\tau}^{\tau+h} S(\tau+h-s) f(s) d s\right) \\
& \leq \frac{1}{h} \int_{\tau}^{\tau+h}\|S(\tau+h-s)(g(s)-f(s))\| d s \leq 2 M e^{\omega(T-\tau)} \varepsilon .
\end{aligned}
$$

Passing to limsup in the inequality above we get

$$
\begin{aligned}
& \limsup _{h \downarrow 0} \frac{1}{h} \operatorname{dist}\left(S(h) \varphi(0)+\int_{\tau}^{\tau+h} S(\tau+h-s) \mathcal{F}_{F(\tau, \varphi)} d s, K(\tau+h)\right) \\
& \leq 2 M e^{\omega(T-\tau)} \varepsilon .
\end{aligned}
$$

As $\varepsilon>0$ was arbitrary, we deduce that $F(\tau, \varphi) \in \mathcal{Q T S}_{\mathcal{K}}^{A}(\tau, \varphi)$.

A simple consequence of Theorem 3.1 is:

Theorem 3.2. If $F: \mathcal{K} \rightsquigarrow X$ is u.s.c. and $\mathcal{K}$ is mild viable with respect to $A+F$ then $F(\tau, \varphi) \in \mathcal{Q T}_{\mathcal{K}}^{A}(\tau, \varphi)$ for all $(\tau, \varphi) \in \mathcal{K}$.

\section{Sufficient conditions for viability}

Definition 4.1. We say that the multi-function $K: I \rightsquigarrow X$ is:

(i) closed from the left on $\mathrm{I}$ if for any sequence $\left(\left(t_{n}, x_{n}\right)\right)_{n \geq 1}$ from $I \times$ $X$, with $x_{n} \in K\left(t_{n}\right)$ and $\left(t_{n}\right)_{n}$ nondecreasing, $\lim _{n} t_{n}=t \in I$ and $\lim _{n} x_{n}=x$, we have $x \in K(t)$.

(ii) locally closed from the left if for each $(\tau, \xi) \in I \times X$ with $\xi \in K(\tau)$ there exist $T>\tau$ and $\rho>0$ such that the multi-function $t \rightsquigarrow K(t) \cap D(\xi, \rho)$ is closed from the left on $[\tau, T]$. 
Definition 4.2. By a Carathéodory uniqueness function we mean a function $\alpha: I \times \mathbb{R}_{+} \rightarrow \mathbb{R}_{+}$such that:

(i) for each $x \in \mathbb{R}_{+}, t \mapsto \alpha(t, x)$ is locally integrable;

(ii) for a.e. $t \in I, x \mapsto \alpha(t, x)$ is continuous, nondecreasing;

(iii) for each $\tau \in I$, the only absolutely continuous solution of the Cauchy problem

$$
\left\{\begin{array}{l}
x^{\prime}(t)=\alpha(t, x(t)) \\
u(\tau)=0
\end{array}\right.
$$

is $x \equiv 0$.

Remark 4.1. If $\alpha: I \times \mathbb{R}_{+} \rightarrow \mathbb{R}_{+}$is a Carathéodory uniqueness function and $x:[\tau, T] \rightarrow X$ is a measurable and bounded function which satisfies

$$
x(t) \leq \int_{\tau}^{t} \alpha(s, x(s)) d s,
$$

for all $t \in[\tau, T]$, then $x(t)=0$ for all $t \in[\tau, T]$.

See Problem 1.8.2. in CÂrJă, Necula and Vrabie [4].

Definition 4.3. We say that $A+F$ is $\beta$-compact if for all $(\tau, \varphi) \in \mathcal{K}$ there exist $\delta>0, \rho>0$, a Carathéodory uniqueness function, $\alpha: I \times$ $\mathbb{R}_{+} \rightarrow \mathbb{R}_{+}$and a continuous function $m:[0, \infty) \rightarrow[0, \infty)$, such that, for all $B \subseteq D_{\sigma}(\varphi, \rho)$, all $t \in(0, \infty)$ and a.e. for $s \in[\tau-\delta, \tau+\delta]$ we have

$$
\lim _{h \downarrow 0} \beta(S(t) F(([s-h, s] \times B) \cap \mathcal{K})) \leq m(t) \alpha(s, \beta(B(0)))
$$

where $B(0)=\{\psi(0) ; \psi \in B\}$.

Remark 4.2. (1) If $\{S(t): X \rightarrow X ; t \geq 0\}$ is compact and $F$ is locally bounded, then $A+F$ is $\beta$-compact;

(2) If $F$ maps bounded subsets of $\mathcal{K}$ into relatively compact subsets of $X$, then $A+F$ is $\beta$-compact;

(3) If $F$ is u.s.c., has compact values and $\mathcal{K}$ is locally compact, then $A+F$ is $\beta$-compact. 
Theorem 4.1. Let $K$ be locally closed from the left and let $F: \mathcal{K} \rightsquigarrow X$ be nonempty, convex and weakly compact valued. If $F$ is strongly-weakly u.s.c., locally bounded and $A+F$ is $\beta$-compact, then a sufficient condition in order that $\mathcal{K}$ be mild viable with respect to $A+F$ is

$$
F(\tau, \varphi) \in \mathcal{Q T S}_{\mathcal{K}}^{A}(\tau, \varphi) \text { for all }(\tau, \varphi) \in \mathcal{K} .
$$

If, in addition, $F$ is u.s.c., then (4.2) is also necessary in order that $\mathcal{K}$ be mild-viable with respect to $A+F$.

The necessity follows from Theorem 3.2, while the sufficiency will be proved later.

From Theorem 4.1, under the additional assumption that $K$ is even closed from the left, by Brezis-Browder Ordering Principle - see BREzIS and BROWDER [1] -, we easily deduce the global mild viability result below.

Theorem 4.2. Let $K$ be closed from the left and let $F: \mathcal{K} \rightsquigarrow X$ be nonempty, convex and weakly compact valued. If $F$ is strongly-weakly u.s.c., locally bounded and $A+F$ is $\beta$-compact, then a sufficient condition in order that $\mathcal{K}$ be globally mild viable with respect to $A+F$ is (4.2).

If, in addition, $F$ is u.s.c., then (4.2) is also necessary in order that $\mathcal{K}$ be mild-viable with respect to $A+F$.

The next lemma, inspired from CÂRJĂ and VRABIE [7], is the main step through the proof of Theorem 4.1.

Lemma 4.1. Let $K: I \rightsquigarrow X$ be locally closed from the left, $F: \mathcal{K} \rightsquigarrow X$ be locally bounded and let $(\tau, \varphi) \in \mathcal{K}$. Let us assume that (4.2) is satisfied.

Let $\rho>0, T>\tau$ and $M_{0}>0$ be such that:

(1) the multi-function $t \rightarrow K(t) \cap D(\varphi(0), \rho)$ is closed from the left on $[\tau, T)$;

(2) $\|F(t, \psi)\| \leq M_{0}$ for all $t \in[\tau, T]$ and all $\psi \in D_{\sigma}(\varphi, \rho)$ with $(t, \psi) \in \mathcal{K}$;

(3) $\sup _{t \in[\tau, T]}\|S(t-\tau) \varphi(0)-\varphi(0)\|+\sup _{|t-s| \leq T-\tau}\|\varphi(t)-\varphi(s)\|+$ $M e^{\omega(T-\tau)}(T-\tau)\left(M_{0}+1\right)<\rho$.

Then, for each $\varepsilon \in(0,1)$, there exist a family $\mathcal{P}_{T}=\left\{\left[t_{m}, s_{m}\right) ; m \in \Gamma\right\}$ of disjoint intervals, with $\Gamma$ finite or at most countable, and four functions: $f, r \in L^{1}(\tau, T ; X), \theta:\{(t, s) ; \tau \leq s \leq t \leq T\} \rightarrow(0, T-\tau]$ measurable, and $u \in C([\tau-\sigma, T] ; X)$ such that: 
(i) $\cup\left[t_{m}, s_{m}\right)=[\tau, T)$ and $s_{m}-t_{m} \leq \varepsilon$, for all $m \in \Gamma$;

(ii) $u\left(t_{m}\right) \in K\left(t_{m}\right)$, for all $m \in \Gamma$ and $u(T) \in K(T)$;

(iii) $\theta(t, s) \leq t-s$ for $\tau \leq s \leq t \leq T$; $t \mapsto \theta(t, s)$ is nonexpansive on $(s, T]$ and, for each $t \in(\tau, T], s \mapsto \theta(t, s)$ is measurable on $[\tau, t)$;

(iv) $f(s) \in F\left(t_{m}, u_{t_{m}}\right)$ a.e. for $s \in\left[t_{m}, s_{m}\right)$ and $\|f(s)\| \leq M_{0}$ a.e. for $s \in[\tau, T]$

(v) $\|r(s)\| \leq \varepsilon$ a.e. for $s \in[\tau, T]$;

(vi) $u(t)=\left\{\begin{array}{l}\varphi(t-\tau), t \in[\tau-\sigma, \tau] \\ S(t-\tau) \varphi(0)+\int_{\tau}^{t} S(t-s) f(s) d s \\ \quad+\int_{\tau}^{t} S(\theta(t, s)) r(s) d s, t \in[\tau, T] ;\end{array}\right.$

(vii) $\left\|u_{t}-\varphi\right\|_{\sigma}<\rho$ for all $t \in[\tau, T]$;

(viii) $\left\|u(t)-u\left(t_{m}\right)\right\| \leq \varepsilon$ for all $t \in\left[t_{m}, s_{m}\right)$ and all $m \in \Gamma$.

Proof. First, let us observe that, if (i) (vi) are satisfied, then (vii) is satisfied too, i.e.

$$
\|u(t+s)-\varphi(s)\|<\rho \text { for all } t \in[\tau, T] \text { and all } s \in[-\sigma, 0] .
$$

Indeed, if $t+s \leq \tau$ then

$$
\|u(t+s)-\varphi(s)\|=\|\varphi(t+s-\tau)-\varphi(s)\| \leq \sup _{\left|t_{1}-t_{2}\right| \leq T-\tau}\left\|\varphi\left(t_{1}\right)-\varphi\left(t_{2}\right)\right\|<\rho .
$$

If $t+s>\tau$ then $|s|<T-\tau$ and from (3) and (vi) we get

$$
\begin{aligned}
& \|u(t+s)-\varphi(s)\| \leq\|S(t+s-\tau) \varphi(0)-\varphi(s)\|+\int_{\tau}^{t}\|S(t-s) f(s)\| d s \\
& +\int_{\tau}^{t}\|S(\theta(t, s)) r(s)\| d s \\
& \leq\|S(t+s-\tau) \varphi(0)-\varphi(0)\|+\|\varphi(0)-\varphi(s)\|+M e^{\omega(T-\tau)} M_{0}(T-\tau) \\
& +M e^{\omega(T-\tau)}(T-\tau)<\rho .
\end{aligned}
$$

Let $\varepsilon \in(0,1)$ be arbitrary, but fixed. We will show that there exist $\delta=\delta(\varepsilon)$ in $(\tau, T)$ and $\mathcal{P}_{\delta}, f, \theta, r, u$ such that $(\mathrm{i}) \sim($ vii) hold true with $\delta$ instead of $T$. 
Using the tangency condition (4.2), we deduce that there exist $h_{n} \downarrow 0$, $g_{n} \in \mathcal{F}_{F(\tau, \varphi)}$ and $p_{n} \in X$, with $\left\|p_{n}\right\| \rightarrow 0$, such that

$$
S\left(h_{n}\right) \varphi(0)+\int_{\tau}^{\tau+h_{n}} S\left(\tau+h_{n}-s\right) g_{n}(s) d s+p_{n} h_{n} \in K\left(\tau+h_{n}\right)
$$

for every $n \in \mathbb{N}, n \geq 1$. Let $n_{0} \in \mathbb{N}$ and $\delta=\tau+h_{n_{0}}$ such that $\delta \in(\tau, T)$, $h_{n_{0}}<\varepsilon$ and $\left\|p_{n_{0}}\right\|<\varepsilon$.

Let us define $\mathcal{P}_{\delta}=\{[\tau, \delta)\}, f(s)=g_{n_{0}}(s), \theta(t, s)=0$ for $\tau \leq s \leq t \leq \delta$, $r(s)=p_{n_{0}}$ for $s \in[\tau, \delta]$ and let $u:[\tau, \delta] \rightarrow X$ given by (vi). One may easily see that (i) $\sim($ vi) are satisfied. Moreover, we may diminish $\delta>\tau$ (increase $n_{0}$ ), if necessary, in order to (viii) be satisfied too.

Let $\mathcal{U}=\left\{\left(\mathcal{P}_{\delta}, f, \theta, r, u\right) ; \delta \in(\tau, T]\right.$ and $(\mathrm{i}) \sim($ vii) are satisfied with $\delta$ instead of $T\}$.

As we already have shown, $\mathcal{U} \neq \emptyset$. On $\mathcal{U}$ we define a partial order by $\left(\mathcal{P}_{\delta_{1}}, f_{1}, \theta_{1}, r_{1}, u_{1}\right) \preceq\left(\mathcal{P}_{\delta_{2}}, f_{2}, \theta_{2}, r_{2}, u_{2}\right)$, if

$$
\left\{\begin{array}{l}
\delta_{1} \leq \delta_{2}, \quad \mathcal{P}_{\delta_{1}} \subseteq \mathcal{P}_{\delta_{2}}, \\
f_{1}(s)=f_{2}(s), r_{1}(s)=r_{2}(s) \text { a.e. for } s \in\left[\tau, \delta_{1}\right], \\
\theta_{1}(t, s)=\theta_{2}(t, s) \text { for } \tau \leq s \leq t \leq \delta_{1}, \\
u_{1}(s)=u_{2}(s) \text { for all } s \in\left[\tau, \delta_{1}\right] .
\end{array}\right.
$$

We will prove that each nondecreasing sequence in $\mathcal{U}$ is bounded from above. Let $\left(\left(\mathcal{P}_{\delta_{j}}, f_{j}, \theta_{j}, r_{j}, u_{j}\right)\right)_{j \geq 1}$ be a nondecreasing sequence in $\mathcal{U}$ and let $\delta=$ $\sup _{j \geq 1} \delta_{j}$. If there exists $j_{0} \in \mathbb{N}$ such that $\delta_{j_{0}}=\delta$, then $\left(\mathcal{P}_{\delta_{j_{0}}}, f_{j_{0}}, \theta_{j_{0}}, r_{j_{0}}, u_{j_{0}}\right)$ is an upper bound for the sequence. So, let us assume that $\delta_{j}<\delta$, for all $j \geq 1$. Obviously, $\delta \in(\tau, T]$. We define $\mathcal{P}_{\delta}=\cup_{j \geq 1} \mathcal{P}_{\delta_{j}}, f(s)=f_{j}(s)$, $\theta(t, s)=\theta_{j}(t, s)$ for $\tau \leq s \leq t \leq \delta_{j}$ and $r(s)=r_{j}(s)$ for all $j$ and all $s \in$ $\left[\tau, \delta_{j}\right)$. Clearly, $f, r \in L^{1}(\tau, \delta ; X)$. Since $\left|\theta_{j}\left(\delta_{j}, s\right)-\theta_{i}\left(\delta_{i}, s\right)\right| \leq\left|\delta_{j}-\delta_{i}\right|$ for all $i, j \geq 1$ and $\tau \leq s<\min \left\{\delta_{i}, \delta_{j}\right\}$, we may define $\theta(\delta, s)=\lim _{j \rightarrow \infty} \theta_{j}\left(\delta_{j}, s\right)$ for all $\tau \leq s<\delta$. It follows that $\theta$ satisfies (iii). Next, we define $u:[\tau, \delta] \rightarrow X$ by

$$
u(t)=S(t-\tau) \varphi(0)+\int_{\tau}^{t} S(t-s) f(s) d s+\int_{\tau}^{t} S(\theta(t, s)) r(s) d s,
$$

for all $t \in[\tau, \delta]$. We have $u \in C([\tau, \delta] ; X)$ and $u(s)=u_{j}(s)$, for all $j \geq 1$ and all $s \in\left[\tau, \delta_{j}\right]$. Since $u(\delta)=\lim _{t \uparrow \delta} u(t)=\lim _{j \rightarrow \infty} u\left(\delta_{j}\right)=$ $\lim _{j \rightarrow \infty} u_{j}\left(\delta_{j}\right)$, and $u_{j}\left(\delta_{j}\right) \in D(\varphi(0), \rho) \cap K\left(\delta_{j}\right)$ and the latter is closed 
from the left, we deduce that $u(\delta) \in D(\varphi(0), \rho) \cap K(\delta)$. The rest of conditions in lemma being obviously satisfied, it follows that $\left(\mathcal{P}_{\delta}, f, \theta, r, u\right)$ is an upper bound for the sequence. Thus, the partially ordered set $(\mathcal{U}, \preceq)$ and the function $\mathcal{N}:(\mathcal{U}, \preceq) \rightarrow R$, defined by $\mathcal{N}\left(\mathcal{P}_{\delta}, f, \theta, r, u\right)=\delta$, for each $\left(\mathcal{P}_{\delta}, f, \theta, r, u\right) \in \mathcal{U}$, satisfy the hypotheses of the Brezis-Browder Ordering Principle, i.e. Theorem 2.1.1, p. 30 in CÂRJĂ, NeCula and VRABIE [4]. Accordingly, there exists an $\mathcal{N}$-maximal element in $\mathcal{U}$. This means that there exists $\left(\mathcal{P}_{\delta^{*}}, f^{*}, \theta^{*}, r^{*}, u^{*}\right) \in \mathcal{U}$ such that, whenever $\left(\mathcal{P}_{\delta^{*}}, f^{*}, \theta^{*}, r^{*}, u^{*}\right) \preceq$ $\left(\mathcal{P}_{\bar{\delta}}, \bar{f}, \bar{\theta}, \bar{r}, \bar{u}\right)$, we necessarily have $\mathcal{N}\left(\mathcal{P}_{\delta^{*}}, f^{*}, \theta^{*}, r^{*}, u^{*}\right)=\mathcal{N}\left(\mathcal{P}_{\bar{\delta}}, \bar{f}, \bar{\theta}, \bar{r}, \bar{u}\right)$. We will show that $\delta^{*}=T$. To this aim, let us assume by contradiction that $\delta^{*}<T$.

Since $\left(\delta^{*}, u_{\delta^{*}}^{*}\right) \in \mathcal{K}$ and using the tangency condition (4.2) we deduce that there exist $h_{n} \downarrow 0, g_{n} \in \mathcal{F}_{F\left(\delta^{*}, u_{\delta^{*}}^{*}\right)}$ and $p_{n} \in X$, with $\left\|p_{n}\right\| \rightarrow 0$, such that

$$
S\left(h_{n}\right) u^{*}\left(\delta^{*}\right)+\int_{\delta^{*}}^{\delta^{*}+h_{n}} S\left(\delta^{*}+h_{n}-s\right) g_{n}(s) d s+h_{n} p_{n} \in K\left(\delta^{*}+h_{n}\right),
$$

for every $n \in \mathbb{N}, n \geq 1$.

Let $n_{0} \in \mathbb{N}$ and $\bar{\delta}=\delta^{*}+h_{n_{0}}$ such that $\bar{\delta} \in\left(\delta^{*}, T\right), h_{n_{0}}<\varepsilon$ and $\left\|p_{n_{0}}\right\|<\varepsilon$. Let us define $\mathcal{P}_{\bar{\delta}}=\mathcal{P}_{\delta^{*}} \cup\left\{\left[\delta^{*}, \bar{\delta}\right]\right\}$,

$\bar{\theta}(t, s)=\left\{\begin{array}{l}\theta^{*}(t, s), \tau \leq s \leq t \leq \delta^{*} \\ t-\delta^{*}+\theta^{*}\left(\delta^{*}, s\right), \tau \leq s<\delta^{*}<t<\bar{\delta} \quad, \\ 0, \delta^{*} \leq s<t \leq \bar{\delta}\end{array}\right.$

$\bar{f}(s)=\left\{\begin{array}{l}f^{*}(s), s \in\left[\tau, \delta^{*}\right] \\ g_{n_{0}}(s), s \in\left(\delta^{*}, \bar{\delta}\right]\end{array} \quad, \bar{r}(s)=\left\{\begin{array}{l}r^{*}(s), s \in\left[\tau, \delta^{*}\right] \\ p_{n_{0}}, s \in\left(\delta^{*}, \bar{\delta}\right]\end{array}\right.\right.$,

$\bar{u}(t)=\left\{\begin{array}{l}u^{*}(t), t \in\left[\tau, \delta^{*}\right] \\ S\left(t-\delta^{*}\right) u^{*}\left(\delta^{*}\right)+\int_{\delta^{*}}^{t} S(t-s) g_{n_{0}}(s) d s+\left(t-\delta^{*}\right) p_{n_{0}}, t \in\left(\delta^{*}, \bar{\delta}\right] .\end{array}\right.$

Since, by (vii) we have $u_{\delta^{*}}^{*} \in S_{\sigma}(\varphi, \rho)$ and using the relation (2), it follows that $\|\bar{f}(s)\| \leq M_{0}$ a.e. for $s \in(\tau, \bar{\delta})$. Clearly (i) $\sim($ vii) are satisfied, and we can diminish $\bar{\delta}$ (increase $n_{0}$ ) in order that (viii) be satisfied too.

So, $\left(\mathcal{P}_{\bar{\delta}}, \bar{f}, \bar{\theta}, \bar{r}, \bar{u}\right) \in \mathcal{U},\left(\mathcal{P}_{\delta^{*}}, f^{*}, \theta^{*}, r^{*}, u^{*}\right) \preceq\left(\mathcal{P}_{\bar{\delta}}, \bar{f}, \bar{\theta}, \bar{r}, \bar{u}\right)$, but $\delta^{*}<\bar{\delta}$ which contradicts the maximality of $\left(\mathcal{P}_{\delta^{*}}, f^{*}, \theta^{*}, r^{*}, u^{*}\right)$. Hence $\delta^{*}=T$, and $\mathcal{P}_{\delta^{*}}, f^{*}, \theta^{*}, r^{*}$ and $u^{*}$ satisfy all the conditions (i) $\sim($ vii). The proof is complete. 
Definition 4.4. Let $\varepsilon>0$. A quintuple $\left(\mathcal{P}_{T}, f, \theta, r, u\right)$ satisfying (i) (viii) in Lemma 4.1, is called an $\varepsilon$-approximate solution of (1.1).

Remark 4.3. Lemma 4.1 offers a sufficient condition in order that, for each $\varepsilon>0$ to exists at least one $\varepsilon$-approximate solution of (1.1).

Next, let us prove Theorem 4.1.

Proof. Since the necessity follows from Theorem 3.2, we will confine ourselves only to the proof of the sufficiency.

Let $\rho>0$ and $T>\tau$ and $M_{0}$ be as in Lemma 4.1. Let $\varepsilon_{n} \in(0,1)$, with $\varepsilon_{n} \downarrow 0$.

Let $\left(\left(\mathcal{P}_{T}^{n}, f_{n}, \theta_{n}, r_{n}, u_{n}\right)\right)_{n}$ be a sequence of $\varepsilon_{n}$-approximate solutions of (1.1), sequence given by Lemma 4.1. If $\mathcal{P}_{T}^{n}=\left\{\left[t_{m}^{n}, s_{m}^{n}\right) ; m \in \Gamma_{n}\right\}$ with $\Gamma_{n}$ finite or at most countable, we denote by $a_{n}:[\tau, T) \rightarrow[\tau, T)$ the step function, defined by $a_{n}(s)=t_{m}^{n}$ for each $s \in\left[t_{m}^{n}, s_{m}^{n}\right)$. Clearly

$$
\lim _{n} a_{n}(s)=s
$$

uniformly for $s \in[\tau, T)$.

In view of (vi), we have

$$
u_{n}(t)=S(t-\tau) \varphi(0)+\int_{\tau}^{t} S(t-s) f_{n}(s) d s+\int_{\tau}^{t} S\left(\theta_{n}(t, s)\right) r_{n}(s) d s
$$

for each $n \in \mathbb{N}$ and $t \in[\tau, T]$. We will show that, on a subsequence at least, $\left(u_{n}\right)_{n}$ is uniformly convergent on $[\tau, T]$ to some function $u$ which will turn out to be a mild solution for the problem (1.1).

We analyze first the case when $X$ is separable.

Using (v) from Lema 4.1 we deduce that, for each $t \in[\tau, T]$, we have

$$
\beta\left(\left\{\int_{\tau}^{t} S\left(\theta_{n}(t, s)\right) r_{n}(s) d s ; n \geq 1\right\}\right)=0 .
$$

From (iv) we get

$$
\left\|f_{n}(t)\right\| \leq M_{0}
$$

for all $n \geq 1$ and a.e. for $t \in[\tau, T]$.

Using (viii) we deduce $\lim _{n}\left\|u_{n}\left(a_{n}(t)\right)-u_{n}(t)\right\|=0$ uniformly for $t \in$ $[\tau, T)$ and from here we get

$$
\beta\left(\left\{u_{n}\left(a_{n}(t)\right) ; n \geq 1\right\}\right)=\beta\left(\left\{u_{n}(t) ; n \geq 1\right\}\right) .
$$


Let $t \in[\tau, T)$. Denote by $x(t)=\beta\left(\left\{u_{n}(t) ; n \geq 1\right\}\right)$ and $B_{t}=\left\{\left(u_{n}\right)_{a_{n}(t)}\right.$; $n \geq 1\}$. By applying the function $\beta$ in (4.4), we obtain

$$
\begin{aligned}
& x(t) \leq \beta\left(\left\{\int_{\tau}^{t} S(t-s) f_{n}(s) d s ; n \geq 1\right\}\right)+\beta\left(\left\{\int_{\tau}^{t} S\left(\theta_{n}(t, s)\right) r_{n}(s) d s ; n \geq 1\right\}\right) \\
& \leq \int_{\tau}^{t} \beta\left(\left\{S(t-s) f_{n}(s) ; n \geq 1\right\}\right) d s .
\end{aligned}
$$

From (iv) in Lemma 4.1, we deduce that, for all $n, k \in \mathbb{N}, n \geq k$ and a.e. for $s \in[\tau, T)$, we have

$$
f_{n}(s) \in F\left(a_{n}(s),\left(u_{n}\right)_{a_{n}(s)}\right) \subset F\left(\left(\left[s-\varepsilon_{n}, s\right] \times B_{s}\right) \cap \mathcal{K}\right) \subset F\left(\left(\left[s-\varepsilon_{k}, s\right] \times B_{s}\right) \cap \mathcal{K}\right)
$$

From here and the fact that $A+F$ is $\beta$-compact we get that, a.e. for $s \in[\tau, t)$

$$
\begin{aligned}
& \beta\left(\left\{S(t-s) f_{n}(s) ; n \geq 1\right\}\right)=\lim _{k \rightarrow \infty} \beta\left(\left\{S(t-s) f_{n}(s) ; n \geq k\right\}\right) \\
& \leq \lim _{k \rightarrow \infty} \beta\left(S(t-s) F\left(\left(\left[s-\varepsilon_{k}, s\right] \times B_{s}\right) \cap \mathcal{K}\right)\right) \leq m(t-s) \alpha\left(s, \beta\left(B_{s}(0)\right)\right) .
\end{aligned}
$$

Let $\alpha_{0}=\left(\sup _{s \in[0, T-\tau]} m(s)\right) \alpha$. It follows that

$$
x(t) \leq \int_{\tau}^{t} \alpha_{0}\left(s, \beta\left(\left\{u_{n}\left(a_{n}(s)\right) ; n \geq 1\right\}\right)\right) d s=\int_{\tau}^{t} \alpha_{0}(s, x(s)) d s .
$$

Since $\alpha_{0}$ is a Carathéodory uniqueness function, too, we deduce that $x(t)=0$ for all $t \in[\tau, T)$. So, $\left\{u_{n}(t) ; n \geq 1\right\}$ is relatively compact for all $t \in[\tau, T)$. By Theorem 8.4.1, p. 194, in VRABIE [19], we conclude that $\left(u_{n}\right)_{n}$ has at least one uniformly convergent subsequence to some function $u$, subsequence denoted, for simplicity, again by $\left(u_{n}\right)_{n}$.

Since $a_{n}(t) \uparrow t, \lim _{n} u_{n}\left(a_{n}(t)\right)=u(t)$, uniformly for $t \in[\tau, T)$ and $t \rightarrow K(t) \cap D(\varphi(0), \rho)$ is closed from the left, we get that $u(t) \in K(t)$ for all $t \in[\tau, T]$.

But $\lim _{n}\left(u_{n}\right)_{a_{n}(t)}=u_{t}$ in $C_{\sigma}$, uniformly for $t \in[\tau, T)$. Hence, the set

$$
C=\overline{\left\{\left(a_{n}(t),\left(u_{n}\right)_{a_{n}(t)}\right) ; n \geq 1, t \in[\tau, T)\right\}}
$$

is compact and $C \subseteq \mathcal{K}$. Since $F$ is strongly-weakly u.s.c. and has weakly compact values, by Lemma 2.6.1, p. 47, in CÂRJĂ, NECUlA and VRABIE [4], it follows that the set

$$
B=\overline{\operatorname{conv}}\left(\bigcup_{n \geq 1} \bigcup_{t \in[\tau, T)} F\left(a_{n}(t),\left(u_{n}\right)_{a_{n}(t)}\right)\right)
$$


is weakly compact. We notice that $f_{n}(s) \in B$ for every $n \geq 1$ and a.e. for $s \in[\tau, T]$, hence, by Diestel's Theorem 1.3.8, p.10, in CÂRJĂ, NECUlA and VRABIE [4], it follows that, on a subsequence at least, $\lim _{n} f_{n}=f$ weakly in $L^{1}(\tau, T ; X)$. As $F$ is strongly-weakly u.s.c. with closed and convex values, and, by Lemma 4.1 , for each $n \geq 1$, we have $f_{n}(s) \in F\left(a_{n}(s),\left(u_{n}\right)_{a_{n}(s)}\right)$ a.e. for $s \in[\tau, T]$, from Theorem 3.1.2, p. 88, in VRABIE [18], we conclude that

$$
f(s) \in F\left(s, u_{s}\right) \text { a.e. for } s \in[\tau, T] .
$$

Finally, passing to the limit both sides in (4.4), for $n \rightarrow \infty$, we get $u(t)=S(t-\tau) \varphi(0)+\int_{\tau}^{t} S(t-s) f(s) d s$, for each $t \in[\tau, T]$.

If $X$ is not separable, we have to observe that there exists a separable and closed subspace $Y \subseteq X$ such that the families: $\left\{S(\cdot) f_{n}(\cdot) ; n \geq 1\right\}$, $\left\{S(\cdot) u_{n}(\cdot) ; n \geq 1\right\}$ and $\left\{S(\cdot) r_{n}(\cdot) ; n \geq 1\right\}$ are $Y$-valued. Then, to complete the proof, it suffices to follows the very same arguments as before and to make use of (iv) in Remark 2.1.

\section{A comparison result}

Let $X$ be a real Banach space, let $C \subset X$ be a closed convex cone and $C \cap(-C)=\{0\}$, let " $\preceq$ " be the partial order on $X$ defined by $C$, i.e., $x \preceq y$ if and only if $y-x \in C$. Let $A: D(A) \subseteq X \rightarrow X$ be the infinitesimal generator of a $C_{0}$-semigroup $\{S(t) ; t \geq 0\}$ and let $a: I \rightarrow X$ be a continuous function. Let $K: I \rightsquigarrow X$ be defined by $K(t)=\{x \in X ; a(t) \preceq x\}$ for each $t \in I$. Let $\mathcal{K}=\left\{(t, \psi) \in I \times C_{\sigma} ; \psi(0) \in K(t)\right\}$ and $F: \mathcal{K} \rightsquigarrow X$ be a given multi-function. We are interested in finding sufficient conditions in order that $\mathcal{K}$ be mild viable with respect to $A+F$, i.e., in order that, for each $(\tau, \varphi) \in \mathcal{K}$, to exists at least one mild solution $u:[\tau, T] \rightarrow X$ of the problem

$$
\left\{\begin{array}{l}
u^{\prime}(t) \in A u(t)+F\left(t, u_{t}\right) \\
u_{\tau}=\varphi \\
a(t) \preceq u(t) \text { for each } t \in[\tau, T] .
\end{array}\right.
$$

The next lemma is, essentially, Lemma 8.1 in Necula, Popescu and Vrabie [16]. See also CÂrjă, Necula and Vrabie [6].

Lemma 5.1. Let $a \in W_{\mathrm{loc}}^{1,1}(I ; X)$ and let $\mathcal{K}$ be as above. Let $\tau \in I$ be a point of differentiability from the right for a, with $a(\tau) \in D(A)$, let $\xi \in C$ and let $E \in \mathcal{B}(X)$. Let us assume that $S(t) C \subset C$ for each $t \geq 0$. Then, the following two conditions: 
(i) $E \in \mathcal{T S}_{\mathcal{K}}^{A}\left(\tau, a_{\tau}+\xi\right)$

(ii) $A a(\tau)-a^{\prime}(\tau)+E \in \mathcal{T S}_{C}^{A}(\xi)($ see relation $(2.5))$

are equivalent. Moreover,

(iii) $\operatorname{dist}\left(A a(\tau)-a^{\prime}(\tau)+E ; C\right)=0$

implies both (i) and (ii).

The next theorem is obtained from Theorem 4.1 and the above lemma.

Theorem 5.1. Let $A: D(A) \subseteq X \rightarrow X$ be the infinitesimal generator of a $C_{0}$-semigroup $\{S(t) ; t \geq 0\}$, let $a: I \rightarrow D(A), a \in C^{1}(I ; X), \mathcal{K}$ be as above and $F: \mathcal{K} \rightsquigarrow X$ be a nonempty, convex and weakly compact valued multi-function. Let us suppose that $S(t) C \subset C$ for every $t>0, F$ is strongly-weakly u.s.c., locally bounded and $A+F$ is $\beta$-compact. Then, any of the next two conditions is a sufficient condition in order that $\mathcal{K}$ be mild viable with respect to $A+F$.

$$
A a(\tau)-a^{\prime}(\tau)+F(\tau, \varphi) \in \mathcal{T S}_{C}^{A}(\varphi(0)-a(\tau))
$$

for all $(\tau, \varphi) \in \mathcal{K}$

$$
\operatorname{dist}\left(A a(\tau)-a^{\prime}(\tau)+F(\tau, \varphi) ; C\right)=0
$$

for all $(\tau, \varphi) \in \mathcal{K}$.

Proof. From Lemma, we deduce that (5.3) implies (5.2) which, in turn, implies that $F(\tau, \varphi) \in \mathcal{T S}_{\mathcal{K}}^{A}\left(\tau, a_{\tau}+\varphi(0)-a(\tau)\right)=\mathcal{T}_{\mathcal{K}}^{A}(\tau, \varphi)$. The conclusion follows from Theorem 4.1.

Remark 5.1. Since $F$ has convex and weakly compact values and $C$ is closed and convex, it results that (5.3) is equivalent with:

for all $(\tau, \varphi) \in \mathcal{K}$, there exists $\eta \in F(\tau, \varphi)$ such that

$$
a^{\prime}(\tau)-A a(\tau) \preceq \eta .
$$

If, in addition, $F$ has compact values, then (5.2) is equivalent with:

$$
\left[A a(\tau)-a^{\prime}(\tau)+F(\tau, \varphi)\right] \cap \mathcal{T}_{C}^{A}(\varphi(0)-a(\tau)) \neq \emptyset
$$

for all $(\tau, \varphi) \in \mathcal{K}$. 


\section{REFERENCES}

1. Brezis, H.; Browder, F. E. - A general principle on ordered sets in nonlinear functional analysis, Advances in Math., 21 (1976), 355-364.

2. Burlic $\breve{A}$, M.; Roşu, D. - A class of nonlinear delay evolution equations with nonlocal initial conditions, Proc. Amer. Math. Soc., in print.

3. Burlică, M.; Roşu, D.; Vrabie, I. I. - Continuity with respect to the data for a delay evolution equation with nonlocal initial conditions, Lib. Math. (N.S.), 32 (2012), $39-50$.

4. CÂrJă, O.; Necula, M.; Vrabie, I. I. - Viability, Invariance and Applications, North-Holland Mathematics Studies, 207, Elsevier Science B.V., Amsterdam, 2007.

5. CÂRJĂ, O.; Necula, M.; VRABIE, I. I. - Necessary and sufficient conditions for viability for semilinear differential inclusions, Trans. Amer. Math. Soc., 361 (2009), 343-390.

6. CÂrJă, O.; Necula, M.; VRabie, I. I. - Tangent sets, viability for differential inclusions and applications, Nonlin. Anal., 71 (2009), e979-e990.

7. CÂrJă, O.; VRABie, I. I. - Some new viability results for semilinear differential inclusions, NoDEA Nonlinear Differential Equations Appl., 4 (1997), 401-424.

8. Gavioli, A.; Malaguti, L. - Viable solutions of differential inclusions with memory in Banach spaces, Portugal. Math., 57 (2000), 203-217.

9. HADDAD, G. - Monotone trajectories of differential inclusions and functionaldifferential inclusions with memory, Israel J. Math., 39 (1981), 83-100.

10. HADDAD, G - Monotone viable trajectories for functional-differential inclusions, J. Differential Equations, 42 (1981), 1-24.

11. Lakshmikantham, V.; Leela, S.; Moauro, V. - Existence and uniqueness of solutions of delay differential equations on a closed subset of a Banach space, Nonlinear Anal., 2 (1978), 311-327.

12. Leela, S.; Moauro, V. - Existence of solutions in a closed set for delay differential equations in Banach spaces, Nonlinear Anal., 2 (1978), 47-58.

13. Lupulescu, V.; Necula, M. - A viability result for nonconvex semilinear functional differential inclusions, Discuss. Math. Differ. Incl. Control Optim., 25 (2005), 109128.

14. MÖNCH, H. - Boundary value problems for nonlinear ordinary differential equations of second order in Banach spaces, Nonlinear Anal., 4 (1980), 985-999. 
15. Necula, M.; Popescu, M.; VRabie, I. I. - Viability for differential inclusions on graphs, Set-Valued Anal., 16 (2008), 961-981.

16. Necula, M.; Popescu, M.; VRABie, I. I. - Evolution equations on locally closed graphs and applications, Nonlinear Anal., 71 (2009), e2205-e2216.

17. PAVEL, N.; IACOB, F. - Invariant sets for a class of perturbed differential equations of retarded type, Israel J. Math., 28 (1977), 254-264.

18. Vrabie, I. I. - Compactness Methods for Nonlinear Evolutions, Second Edition. Pitman Monographs and Surveys in Pure and Applied Mathematics, 75, Longman, 1995.

19. VRabie, I. I. - $C_{0}$-semigroups and Applications, North-Holland Mathematics Studies, 191, North-Holland Publishing Co., Amsterdam, 2003.

20. VRABIE, I. I. - Existence for nonlinear evolution inclusions with nonlocal retarded initial conditions, Nonlinear Anal., 74 (2011), 7047-7060.

21. VRabie, I. I. - Existence in the large for nonlinear delay evolution inclusions with nonlocal initial conditions, J. Funct. Anal., 262 (2012), 1363-1391.

22. VRABIE, I. I. - Global solutions for nonlinear delay evolution inclusions with nonlocal initial conditions, Set-Valued Var. Anal., 20 (2012), 477-497.

23. VRABIE, I. I. - Nonlinear retarded evolution equations with nonlocal initial conditions, Dynam. Systems Appl., 21 (2012), 417-440.

Received: 1.XI.2012

Accepted: 1.II.2013
Faculty of Mathematics, "Al. I. Cuza" University, Iaşi 700506, ROMANIA necula@uaic.ro

Department of Mathematics, "Dunărea de Jos" University, Galaţi, 800201, ROMANIA Marius.Popescu@ugal.ro 